\title{
Theoretical Performance Analysis of Single and Multiple Antenna BFWA Systems
}

\author{
Pei Xiao, Rolando Carrasco \\ School of Electrical, Electronic and Computer Engineering \\ University of Newcastle Upon Tyne NE1 7RU, United Kingdom \\ E-mail: pei.xiao, r.carrascoencl.ac.uk \\ Ian Wassell \\ Computer Laboratory, University of Cambridge \\ 15 JJ Thomson Avenue, CB3 OFD, United Kingdom \\ E-mail: ijw24@cam.ac.uk
}

\begin{abstract}
The systems under investigation are broadband fixed wireless access (BFWA) systems operating in multipath fading channels. Conventional detection methods, e.g., coherent detection for single-input, single-output (SISO) systems and the Alamouti algorithm for multiple-input, multiple-output (MIMO) systems are examined theoretically in this paper and shown to yield unsatisfactory performance. The theoretical analyses are validated by Monte-Carlo simulations and are demonstrated to be accurate. The asymptotic performance of the space-time block coded (STBC) system with the Alamouti transmission scheme is also evaluated. However, the results indicate that the performance lower bound cannot be obtained in an uncoded system due to the error propagation problem, which can be tackled by concatenating the STBC system with an outer channel code and applying the turbo processing principle. The theoretical performance analysis conducted in this paper give us an insight into the physical limitations imposed by the BFWA channels and suggest solutions to improve the capacity and performance of future BFWA systems.
\end{abstract}

Keywords: broadband fixed wireless access, theoretical analysis, multipath fading channels, SISO, MIMO. 


\section{INTRODUCTION}

It is becoming apparent that access to the Internet is of growing economic and political importance. It is also clear that low bandwidth dial-up Internet access is restricting the services and applications that can be offered. What is required is a quantum leap in access bandwidth to free up the Internet for innovative applications. One possible solution is to use the existing local-loop. This approach requires the installation of digital subscriber line (DSL) equipment at the exchange and customer premises. Unfortunately, the length and quality of the local-loop infrastructure will prevent this service being offered universally. Another option for providing broadband access is via cable TV networks. However, the availability of these services is far from universal and contention will seriously degrade throughput per user. An alternative approach is to deploy broadband fixed wireless access (BFWA) technology. The advantage of such an approach is that it enables operators in a competitive environment to roll-out broadband services in a rapid and cost efficient manner. BFWA networks generally employ a point-to-multi-point architecture, where a single base station (BS) communicates with many subscriber units (SUs) placed at the user locations. Standardization of BFWA systems is currently being undertaken by the IEEE 802.16 working group [1] and the ETSI HIPERMAN group [2]. Both orthogonal frequency division multiplexing (OFDM) and single-carrier solutions have been adopted in IEEE 802.16 standard as two alternatives for BFWA systems operating at 2-11 GHz bands [3]. An overview of BFWA technology, the characteristics of BFWA channels, and a description of the physical layer specifications can be found in [4], [5]. One of the limiting factors in outdoor wireless transmission is the multipath channel between the transmitter and the receiver. This gives rise to intersymbol interference (ISI), which degrades the system performance and limits the maximum achievable data rate. To our best knowledge, the effect of ISI on the performance of the BFWA systems has not been investigated theoretically in the existing literature. In this paper, we provide a theoretical approach to analyze the the performance of BFWA single-input, single-output (SISO) and multiple-input, multiple-output (MIMO) systems as well as the asymptotic performance of the space-time block coded (STBC) BFWA systems under idealistic conditions with the aim of gaining a deep insight into the physical limitations imposed by BFWA channels on conventional detection techniques and the theoretical capacity of the systems. The asymptotic study leads to a feasible solution to overcome the limitations of the BFWA channels. The main contributions of this paper are outlined as follows: 1) A closed form expression of the average bit error probability for coherent detection in the BFWA SISO system is derived. 2) A theorectical analysis of the performance of the Alamouti algorithm and its modified version (with interference cancellation and multipath combining) is conducted; the bit error probablitis of these schemes for BFWA MIMO systems are derived and compared to the simulation results. 3) The asymptotic performance of the STBC BFWA systems with the Alamouti transmission scheme is evaluated and the asympototic study suggests the design of a space-time turbo equalization scheme that can improve the capacity and performance of BFWA systems. 
The multipath channel can be modeled by an equivalent baseband system where the transmit filter, the channel and the receive filter, are represented by a discrete-time $L$-tap transversal filter with finite-

length impulse response $h_{n}=\sum_{l=0}^{L-1} h_{l} \delta_{n-l}$ where $h_{l}$ denotes the complex channel coefficients. Tailored for different terrain conditions, a set of 6 typical channel models, known as the Stanford University Interim (SUI) Channel Models have been proposed in [6] for simulation, design, development and testing of BFWA systems. All of the SUI models have 3 taps, having either Rayleigh or Ricean amplitude distributions. For the purpose of this study, we have chosen the SUI-3 channel which has a tap spacing of 500ns, and maximum tap delay of 1000ns. To simplify the analysis, we assume the transmitted symbol rate is $2 \mathrm{M}$ symbols/s yielding a data rate of $4 \mathrm{Mbps}$ with QPSK modulation. In this case, the multipath fading is modeled as a tapped-delay line with adjacent taps spaced equally at the symbol duration. The received signal for a SISO system is formed as

$$
r_{n}=h_{0} s_{n}+h_{1} s_{n-1}+h_{2} s_{n-2}+v_{n}
$$

where the channel coefficients $h_{0}, h_{1}, h_{2}$ are complex Gaussian distributed and assumed to remain constant during the transmission of one block of data. They, however, vary from block to block. The amplitude of the first tap $\left|h_{0}\right|$ is characterized by a Ricean distribution due to the line of sight (LOS) propagation. The amplitudes of the taps $\left|h_{1}\right|,\left|h_{2}\right|$ are Rayleigh distributed since they are not owing to LOS propagation. The transmitted symbol at time instant $n$ is denoted as $s_{n}=x_{n}+j y_{n}$, and $v_{n}$ is the complex additive white Gaussian noise with zero mean and variance $N_{0}$.

\section{Performance of Coherent Detection for Siso System}

Let us denote $\hat{h}_{0}$ as an estimate of $h_{0}$, and assume it is an accurate estimate, i.e., $\hat{h}_{0} \approx h_{0}$. To detect the transmitted symbols coherently, we multiply the received signal with the conjugate of $\hat{h}_{0}$, i.e.,

$$
\begin{aligned}
r_{n}^{\prime} & =\hat{h}_{0}^{*}\left(h_{0} s_{n}+h_{1} s_{n-1}+h_{2} s_{n-2}+v_{n}\right)=\hat{h}_{0}^{*} h_{0} s_{n}+\underbrace{\hat{h}_{0}^{*}\left(h_{1} s_{n-1}+h_{2} s_{n-2}+v_{n}\right)}_{\text {combined ISI and noise }} \\
& =\hat{h}_{0}^{*} h_{0} s_{n}+w_{n} \approx\left|h_{0}\right|^{2} s_{n}+w_{n},
\end{aligned}
$$

where the superscript operator ()$^{*}$ is the conjugate transpose operation when applied to matrices and vectors, and simply the conjugate when applied to scalars. The combined intersymbol interference (ISI) and noise $w_{n}=w_{I}+j w_{Q}$ is a complex Gaussian random variable with PDF $w_{n} \sim \mathcal{C N}\left(0, N_{w}\right)$ and variance

$$
N_{w}=\left|h_{0}\right|^{2}\left(\mathrm{E}\left[\left|h_{1}\right|^{2}\right]+\mathrm{E}\left[\left|h_{2}\right|^{2}\right]+N_{0}\right)=\left|h_{0}\right|^{2}\left(P_{1}+P_{2}+N_{0}\right)
$$

In the following, we shall analyze the performance of coherent detection for the BFWA system with QPSK modulation. The QPSK signal constellation is plotted in Fig. 1. With maximum likelihood detection, the optimum decision regions are simply four quadrants $R_{0}, R_{1}, R_{2}, R_{3}$. The probability of 
making a symbol error, e.g., $\hat{s}_{n}=s_{1}$ given $s_{0}$ is transmitted (i.e., $s_{n}=s_{0}$ ) is the probability of $r_{n}^{\prime}$ falling in the decision region $R_{1}$, i.e.,

$$
\begin{aligned}
& P\left(\hat{s}_{n}=s_{1} \mid s_{n}=s_{0}\right)=P_{r}\left\{r_{n}^{\prime}=\left|h_{0}\right|^{2} s_{0}+w_{n} \in R_{1}\right\} \\
& =P_{r}\left\{A\left|h_{0}\right|^{2}+w_{I}+j\left(A\left|h_{0}\right|^{2}+w_{Q}\right) \in R_{1}\right\}=P_{r}\left\{w_{I}<-A\left|h_{0}\right|^{2}\right\} \cdot P_{r}\left\{w_{Q}>-A\left|h_{0}\right|^{2}\right\},
\end{aligned}
$$

The third equality in (4) holds since the Gaussian random variables $w_{I} \sim \mathcal{N}\left(0, N_{w} / 2\right)$ and $w_{Q} \sim$ $\mathcal{N}\left(0, N_{w} / 2\right)$ are statistically independent. Normalizing $w_{I}, w_{Q}$ to unit variance, yields

$$
\begin{aligned}
& P\left(\hat{s}_{n}=s_{1} \mid s_{n}=s_{0}\right)=P_{r}\left\{\frac{w_{I}}{\sqrt{N_{w} / 2}}<-\frac{A\left|h_{0}\right|^{2}}{\sqrt{N_{w} / 2}}\right\} \cdot P_{r}\left\{\frac{w_{Q}}{\sqrt{N_{w} / 2}}>-\frac{A\left|h_{0}\right|^{2}}{\sqrt{N_{w} / 2}}\right\} \\
& =Q\left(\sqrt{\frac{2 E_{b}\left|h_{0}\right|^{2}}{P_{1}+P_{2}+N_{0}}}\right)\left[1-Q\left(\sqrt{\frac{2 E_{b}\left|h_{0}\right|^{2}}{P_{1}+P_{2}+N_{0}}}\right)\right],
\end{aligned}
$$

where $Q(x)=\int_{x}^{\infty} \frac{1}{\sqrt{2 \pi}} \exp \left(-t^{2} / 2\right) d t$ is the complementary Gaussian cumulative distribution function. The above equation holds since $E_{s}=2 A^{2}$, and the bit energy is half of the symbol energy for QPSK modulation, i.e., $E_{b}=E_{s} / 2$. Similarly, other conditional error probabilities can be obtained as

$$
\begin{aligned}
& P\left(\hat{s}_{n}=s_{2} \mid s_{n}=s_{0}\right)=Q^{2}\left(\sqrt{\frac{2 E_{b}\left|h_{0}\right|^{2}}{P_{1}+P_{2}+N_{0}}}\right) \\
& P\left(\hat{s}_{n}=s_{3} \mid s_{n}=s_{0}\right)=Q\left(\sqrt{\frac{2 E_{b}\left|h_{0}\right|^{2}}{P_{1}+P_{2}+N_{0}}}\right)\left[1-Q\left(\sqrt{\frac{2 E_{b}\left|h_{0}\right|^{2}}{P_{1}+P_{2}+N_{0}}}\right)\right],
\end{aligned}
$$

According to the signal constellation shown in Fig. 1, the error event $\left(\hat{s}_{n}=s_{2} \mid s_{n}=s_{0}\right)$ results in 2 bits in error, while both $\left(\hat{s}_{n}=s_{1} \mid s_{n}=s_{0}\right)$ and $\left(\hat{s}_{n}=s_{3} \mid s_{n}=s_{0}\right)$ result in 1 bit in error. The relationship between bit error probability and the conditional symbol error probabilities is therefore

$$
P_{b}=\frac{1}{2}\left[2 \times P\left(\hat{s}_{n}=s_{2} \mid s_{n}=s_{0}\right)+1 \times P\left(\hat{s}_{n}=s_{1} \mid s_{n}=s_{0}\right)+1 \times P\left(\hat{s}_{n}=s_{3} \mid s_{n}=s_{0}\right)\right],
$$

where the factor $\frac{1}{2}$ is due to the fact that one QPSK symbol corresponds to 2 bits. Based on (5), (6) and (7), the bit error probability is derived as

$$
P_{b}(r)=Q\left(\sqrt{\frac{2 E_{b}\left|h_{0}\right|^{2}}{P_{1}+P_{2}+N_{0}}}\right)=Q\left(\frac{\sqrt{2 E_{b}} r}{\sqrt{P_{1}+P_{2}+N_{0}}}\right) .
$$

In (8), we denote $r=\left|h_{0}\right|$, which is Ricean distributed with PDF

$$
p(r)=\frac{r}{\sigma^{2}} \exp \left(-\frac{r^{2}+s^{2}}{2 \sigma^{2}}\right) I_{0}\left(\frac{r s}{\sigma^{2}}\right), \quad r \geq 0,
$$

where $I_{0}(x)$ is the $0^{\text {th }}$ order modified Bessel function of the first kind [7, p. 44]

$$
\begin{gathered}
I_{0}(x)=\sum_{k=0}^{\infty} \frac{(x / 2)^{2 k}}{k ! \Gamma(k+1)}=\sum_{k=0}^{\infty} \frac{(x / 2)^{2 k}}{(k !)^{2}}, \quad x \geq 0, \\
I_{0}\left(\frac{r s}{\sigma^{2}}\right)=\sum_{k=0}^{\infty} \frac{(r s)^{2 k}}{\left(2 \sigma^{2}\right)^{2 k}(k !)^{2}}=\sum_{k=0}^{\infty} \frac{(r s)^{2 k}}{4^{k} \sigma^{4 k}(k !)^{2}}, \quad r \geq 0 .
\end{gathered}
$$


To obtain the error probability when $r$ is random, we must average $P_{b}(r)$ given in (8) over the distribution of $r$, i.e.,

$$
\begin{aligned}
\bar{P}_{b} & =\int_{0}^{\infty} P_{b}(r) p(r) d r=\int_{0}^{\infty} Q\left(\frac{\sqrt{2 E_{b}} r}{\sqrt{P_{1}+P_{2}+N_{0}}}\right) \frac{r}{\sigma^{2}} \exp \left(-\frac{r^{2}+s^{2}}{2 \sigma^{2}}\right) \sum_{k=0}^{\infty} \frac{(r s)^{2 k}}{4^{k} \sigma^{4 k}(k !)^{2}} d r \\
& =\frac{\exp \left(-s^{2} / 2 \sigma^{2}\right)}{\sigma^{2}} \int_{0}^{\infty} Q\left(\frac{\sqrt{2 E_{b}} r}{\sqrt{P_{1}+P_{2}+N_{0}}}\right) r \exp \left(-\frac{r^{2}}{2 \sigma^{2}}\right) \sum_{k=0}^{\infty} \frac{(r s)^{2 k}}{4^{k} \sigma^{4 k}(k !)^{2}} d r .
\end{aligned}
$$

Assigning $x=r / \sigma$ or $r=x \sigma$, and $k=n-1$, equation (11) becomes

$$
\begin{aligned}
\bar{P}_{b} & =\frac{\exp \left(-s^{2} / 2 \sigma^{2}\right)}{\sigma^{2}} \int_{0}^{\infty} Q\left(\frac{\sqrt{2 E_{b}} \sigma x}{\sqrt{P_{1}+P_{2}+N_{0}}}\right) \sigma x \exp \left(-\frac{x^{2}}{2}\right) \sum_{k=0}^{\infty} \frac{(\sigma x s)^{2 k}}{4^{k} \sigma^{4 k}(k !)^{2}} d(\sigma x) \\
& =\exp \left(\frac{-s^{2}}{2 \sigma^{2}}\right) \int_{0}^{\infty} Q\left(\frac{\sqrt{2 E_{b}} \sigma x}{\sqrt{P_{1}+P_{2}+N_{0}}}\right) \exp \left(-\frac{x^{2}}{2}\right) \sum_{k=0}^{\infty} \frac{(\sigma s)^{2 k} x^{2 k+1}}{4^{k} \sigma^{4 k}(k !)^{2}} d x \\
& =\exp \left(\frac{-s^{2}}{2 \sigma^{2}}\right) \int_{0}^{\infty} Q\left(\frac{\sqrt{2 E_{b}} \sigma x}{\sqrt{P_{1}+P_{2}+N_{0}}}\right) \exp \left(-\frac{x^{2}}{2}\right) \sum_{n=1}^{\infty} \frac{(\sigma s)^{2 n-2} x^{2 n-1}}{4^{n-1} \sigma^{4 n-4}[(n-1) !]^{2}} d x .
\end{aligned}
$$

According to [8, p. 102],

$$
\int_{0}^{\infty} x^{2 n-1} \exp \left(-\frac{x^{2}}{2}\right) Q\left(\frac{x}{\alpha}\right) d x=\frac{(n-1) !}{2}(1-\beta)^{n} \sum_{k=0}^{n-1} 2^{-k}\left(\begin{array}{c}
n-1+k \\
k
\end{array}\right)(1+\beta)^{k},
$$

where $\beta=\left(\alpha^{2}+1\right)^{-1 / 2}$. Consequently, equation (12) can be reformed as

$$
\begin{aligned}
\bar{P}_{b} & =\exp \left(\frac{-s^{2}}{2 \sigma^{2}}\right) \sum_{n=1}^{\infty} f(n) \frac{(n-1) !}{2}(1-\beta)^{n} \sum_{k=0}^{n-1} 2^{-k}\left(\begin{array}{c}
n-1+k \\
k
\end{array}\right)(1+\beta)^{k} \\
& \approx \frac{1}{2} \exp \left(\frac{-s^{2}}{2 \sigma^{2}}\right) \sum_{n=1}^{N} g(n)(1-\beta)^{n} \sum_{k=0}^{n-1} 2^{-k}\left(\begin{array}{c}
n-1+k \\
k
\end{array}\right)(1+\beta)^{k}
\end{aligned}
$$

where

$f(n)=\frac{(\sigma s)^{2 n-2}}{4^{n-1} \sigma^{4 n-4}[(n-1) !]^{2}} ; \quad g(n)=\frac{(\sigma s)^{2 n-2}}{4^{n-1} \sigma^{4 n-4}(n-1) !} ; \quad \alpha=\sqrt{\frac{P_{1}+P_{2}+N_{0}}{2 E_{b} \sigma^{2}}} ; \quad \beta=\left(\alpha^{2}+1\right)^{-1 / 2}$

The approximation in (14) is due to the fact that for a sufficient high value of $n$, say $n>N$, the term $\mathcal{J}(n)=g(n)(1-\beta)^{n} \sum_{k=0}^{n-1} 2^{-k}\left(\begin{array}{c}n-1+k \\ k\end{array}\right)(1+\beta)^{k}$ will be negligible, and we can thus truncate the sum without a significant loss of accuracy. The truncation length $N$ can be determined according to some criterion, e.g., by choosing the smallest integer which satisfies the condition $\mathcal{J}(N)<10^{-6}$.

\section{Performance of the Alamouti Algorithm for Mimo Systems}

As stated in [4], the major challenge when designing a BFWA system is to provide high-data-rate wireless access at almost wire-line quality. The high quality of service requirement arises because wireless BFWA systems have to compete with cable modems and ADSL which operate over wired channels and hence provide very good quality. The use of multiple antennas at the transmitter and receiver sides of a wireless link in combination with signal processing and coding is an effective means to satisfy quality of service requirements. Space-time codes were first introduced in [9] to provide transmit diversity in wireless fading channels using multiple antennas. There are two main types of space-time codes, namely, 
space-time trellis codes (STTC) [9] and space-time block codes (STBC) [10], [11]. The performance of STTC and STBC is compared in [12]. The advantage of STTCs over STBCs is the provision of coding gain, however, this is achieved at the cost of high complexity encoders and decoders. For the purpose of this work, we consider the use of STBC, in particular, the two-antenna transmit diversity scheme [10] proposed by Alamouti.

Each complex channel coefficient is denoted as $h_{i j}^{l}$ where the first (second) subscript $i(j)$ is the index of the transmit (receive) antenna, the superscript $l$ refers to the number of the channel tap. For example, $h_{10}^{0}$ denotes the channel coefficient corresponding to the first tap of the channel between the transmit antenna $t x 1$ and the receive antenna $r x 0$. The channel coefficients are complex Gaussian random variables. The amplitude of the first tap $\left|h_{i j}^{0}\right|$ is characterized by a Ricean distribution owing to the presence of LOS propagation. The amplitudes of the other two taps $\left|h_{i j}^{1}\right|,\left|h_{i j}^{2}\right|$ are Rayleigh distributed since these two paths are not owing to LOS propagation. The encoding and transmission sequence of the information symbols is shown in Table I. The information bits $\left\{b_{n}\right\}$ are mapped into QPSK symbols $\left\{s_{n}\right\}$. The transmitted symbols are grouped into blocks of 2 symbols at each antenna. At a given time, two symbols are simultaneously transmitted from the two antennas. At time instance $t$, the symbol transmitted from antenna zero is denoted as $s_{n}^{0}$, and the symbol transmitted from antenna one is denoted as $s_{n}^{1}$. During the next symbol period $t+T$, symbol $-s_{n}^{1 *}$ is transmitted from antenna zero, and $s_{n}^{0 *}$ is transmitted from antenna one. The two transmit antennas and one receive antenna (2TX-1RX) system will now be analyzed. The received signals at antenna $r x 0$ during these two symbol periods can be formed as

$$
\begin{aligned}
& r_{n}^{0}=h_{00}^{2} s_{n-1}^{0}-h_{00}^{1} s_{n-1}^{1 *}+\underline{h_{00}^{0} s_{n}^{0}}+h_{10}^{2} s_{n-1}^{1}+h_{10}^{1} s_{n-1}^{0 *}+\underline{h_{10}^{0} s_{n}^{1}}+v_{n}^{0} \\
& r_{n}^{1}=-h_{00}^{2} s_{n-1}^{1 *}+h_{00}^{1} s_{n}^{0}-\underline{h_{00}^{0} s_{n}^{1 *}+h_{10}^{2} s_{n-1}^{0 *}+h_{10}^{1} s_{n}^{1}+\underline{h_{10}^{0} s_{n}^{0 *}}+v_{n}^{1} .}
\end{aligned}
$$

where $v_{n}^{0}\left(v_{n}^{1}\right)$ is the complex additive white Gaussian noise with zero mean and variance $N_{0}$. The desired symbols in the above equations are underlined so that they can be distinguished from the interference symbols. According to the original Alamouti algorithm, the soft decisions on symbols $s_{n}^{0}, s_{n}^{1}$ can be formed as

$$
\begin{aligned}
\widetilde{s_{n}^{0}} & =h_{00}^{0 *} r_{n}^{0}+h_{10}^{0} r_{n}^{1 *}=\underline{\left|h_{00}^{0}\right|^{2} s_{n}^{0}}+h_{00}^{0 *} h_{10}^{0} s_{n}^{1}+h_{00}^{0 *}\left(h_{00}^{2} s_{n-1}^{0}-h_{00}^{1} s_{n-1}^{1 *}+h_{10}^{2} s_{n-1}^{1}+h_{10}^{1} s_{n-1}^{0 *}+v_{n}^{0}\right) \\
& +\underline{\left|h_{10}^{0}\right|^{2} s_{n}^{0}}-h_{10}^{0} h_{00}^{0 *} s_{n}^{1}+h_{10}^{0}\left(-h_{00}^{2 *} s_{n-1}^{1}+h_{00}^{1 *} s_{n}^{0 *}+h_{10}^{2 *} s_{n-1}^{0}+h_{10}^{1 *} s_{n}^{1 *}+v_{n}^{1 *}\right) \\
& =\left(\left|h_{00}^{0}\right|^{2}+\left|h_{10}^{0}\right|^{2}\right) s_{n}^{0}+w_{n}^{0}=\gamma^{0} s_{n}^{0}+w_{n}^{0} \\
\widetilde{s_{n}^{1}} & =h_{10}^{0 *} r_{n}^{0}-h_{00}^{0} r_{n}^{1 *}=\ldots=\left(\left|h_{00}^{0}\right|^{2}+\left|h_{10}^{0}\right|^{2}\right) s_{n}^{1}+w_{n}^{1}=\gamma^{0} s_{n}^{1}+w_{n}^{1}
\end{aligned}
$$

where $\gamma^{0}=\left|h_{00}^{0}\right|^{2}+\left|h_{10}^{0}\right|^{2}$, and $w_{n}^{0}\left(w_{n}^{1}\right)$ denotes the combined ISI and noise, and is a complex Gaussian random variable with $\operatorname{PDF} w_{n}^{0}\left(w_{n}^{1}\right) \sim \mathcal{C N}\left(0, N_{w}\right)$, and variance

$$
N_{w}=\left(\left|h_{00}^{0}\right|^{2}+\left|h_{10}^{0}\right|^{2}\right)\left(P_{00}^{1}+P_{10}^{1}+P_{00}^{2}+P_{10}^{2}+N_{0}\right)=\gamma^{0}\left(P_{00}^{1}+P_{10}^{1}+P_{00}^{2}+P_{10}^{2}+N_{0}\right),
$$


where $P_{i j}^{l}=\mathrm{E}\left[\left|h_{i j}^{l}\right|^{2}\right]$. Following the same procedure as in Section II, the conditional bit error probability is

$$
P_{b}\left(\gamma^{0}\right)=Q\left(\sqrt{\frac{2 E_{b} \gamma^{0}}{P_{00}^{1}+P_{10}^{1}+P_{00}^{2}+P_{10}^{2}+N_{0}}}\right) .
$$

The average bit error probability is obtained by averaging $P_{b}\left(\gamma^{0}\right)$ over the distributions of $\gamma^{0}$, i.e., $\bar{P}_{b}=\int_{\gamma^{0}} P_{b}\left(\gamma^{0}\right) p\left(\gamma^{0}\right) d \gamma^{0}$, where $p\left(\gamma^{0}\right)$ is PDF function of the random variable $\gamma^{0}$. Deriving $p\left(\gamma^{0}\right)$ is straightforward if $h_{00}^{0}$ and $h_{10}^{0}$ are not correlated. However, the SUI channel models also define an antenna correlation coefficient, which has to be taken into considerations for multiple antenna BFWA channels. Antenna correlation is defined as the envelope correlation coefficient between signals received at an antenna element, i.e.,

$$
\rho=\left|\frac{\mathrm{E}\left\{\left(h_{i j}^{l}-E\left\{h_{i j}^{l}\right\}\right)\left(h_{m n}^{l}-E\left\{h_{m n}^{l}\right\}\right)^{*}\right\}}{\sqrt{\mathrm{E}\left\{\left|h_{i j}^{l}-E\left\{h_{i j}^{l}\right\}\right|^{2}\right\} \mathrm{E}\left\{\left|h_{m n}^{l}-E\left\{h_{m n}^{l}\right\}\right|^{2}\right\}}}\right|=\mid \frac{\operatorname{cov}\left(h_{i j}^{l}, h_{m n}^{l}\right)}{\sqrt{\operatorname{var}\left(h_{i j}^{l}\right) \operatorname{var}\left(h_{m n}^{l}\right)}},,
$$

where $i, j, m, n \in\{0,1\}, l \in\{0,1,2\}$. According to the IEEE802.16 specification [1], the taps with different delays are uncorrelated within a channel as well as between channels, i.e., $\mathrm{E}\left[h_{i j}^{k} h_{m n}^{p *}\right]=0$, for $k \neq p$.

Finding $p\left(\gamma^{0}\right)$ is tedious in presence of antenna correlation (i.e., when $h_{00}^{0}$ and $h_{10}^{0}$ are not statistically independent). To work around this problem, we form a channel vector $\mathbf{h}^{0}=\left[\begin{array}{ll}h_{00}^{0} & h_{10}^{0}\end{array}\right]^{T}$, where ${ }^{T}$ denotes the transpose operation. The joint PDF of $\mathbf{h}^{0}$ is determined by its mean vector $\mathbf{m}_{\mathbf{h}}^{0}$ and its covariance matrix $\mathbf{C}_{\mathbf{h}}^{0}=\mathrm{E}\left\{\left[\mathbf{h}^{0}-\mathbf{m}_{\mathbf{h}}^{0}\right]\left[\mathbf{h}^{0}-\mathbf{m}_{\mathbf{h}}^{0}\right]^{*}\right\}$, i.e.,

$$
p\left(\mathbf{h}^{0}\right)=\frac{1}{(2 \pi)^{2}\left(\operatorname{det} \mathbf{C}_{\mathbf{h}}^{0}\right)^{1 / 2}} \exp \left[-\frac{1}{2}\left(\mathbf{h}^{0}-\mathbf{m}_{\mathbf{h}}^{0}\right)^{*} \mathbf{C}_{\mathbf{h}}^{0-1}\left(\mathbf{h}^{0}-\mathbf{m}_{\mathbf{h}}^{0}\right)\right] .
$$

Note that the antenna correlation is taken into account in the covariance matrix $\mathbf{C}_{\mathbf{h}}^{0}$. Since $\gamma^{0}=\mathbf{h}^{0 *} \mathbf{h}^{0}$, the conditional and the average bit error probabilities can be expressed as

$$
P_{b}\left(\mathbf{h}^{0}\right)=Q\left[\sqrt{\frac{2 E_{b} \mathbf{h}^{0 *} \mathbf{h}^{0}}{P_{00}^{1}+P_{10}^{1}+P_{00}^{2}+P_{10}^{2}+N_{0}}}\right] ; \quad \bar{P}_{b}=\int_{\mathbf{h}^{0}} P_{b}\left(\mathbf{h}^{0}\right) p\left(\mathbf{h}^{0}\right) d \mathbf{h}^{0} .
$$

The conventional definition of the Q-function is given by $Q(x)=\int_{x}^{\infty} \frac{1}{\sqrt{2 \pi}} \exp \left(-\frac{y^{2}}{2}\right) d y$, which is substituted into (21) to derive the average bit error probability. However, the analysis is difficult to perform in this way since the argument $x$ appears in the lower limit of the integral. The problem can be tackled by using an alternative definite integral form for the Q-function [13]

$$
Q(x)=\frac{1}{\pi} \int_{0}^{\pi / 2} \exp \left(-\frac{x^{2}}{2 \sin ^{2} \theta}\right) d \theta .
$$

Substituting (22) into (21), $\bar{P}_{b}$ can be obtained according to [14] as

$$
\bar{P}_{b}=\frac{1}{\pi} \int_{0}^{\frac{\pi}{2}}\left[\operatorname{det}\left(\frac{\boldsymbol{\Sigma}^{0}}{\sin ^{2} \theta}+\mathbf{I}\right)\right]^{-1} \exp \left[-\mathbf{m}^{0 *}\left(\boldsymbol{\Sigma}^{0}+\sin ^{2} \theta \mathbf{I}\right)^{-1} \mathbf{m}^{0}\right] d \theta,
$$

where $\mathbf{m}^{0}=\mathbf{m}_{h}^{0} / \alpha, \boldsymbol{\Sigma}^{0}=\mathbf{C}_{\mathbf{h}}^{0} / \alpha^{2}, \alpha=\sqrt{\left(P_{00}^{1}+P_{10}^{1}+P_{00}^{2}+P_{10}^{2}+N_{0}\right) / E_{b}}$. This single finite-range integral can easily be evaluated numerically although it is not a closed form expression.

The performance analysis for 2TX-2RX system can be carried out similarly, but for brevity it will not be presented here. 


\section{Analysis of the Asymptotic Performance}

The Alamouti algorithm was originally developed for flat fading channels and so does not take into consideration the ISI introduced by frequency-selective fading channels. As we will see in Section V, it results in very poor performance for the BFWA channels. An equalizer is an effective remedy to combat the detrimental effects caused by ISI. Equalization for STBC coded systems has been treated in several papers, e.g., in [15], where a zero-forcing (ZF) and minimum mean-square error (MMSE) schemes were presented to combat ISI and obtain diversity gain. However, both ZF and MMSE equalizers involve a matrix inversion at symbol rate, which significantly increases the complexity at the receiver compared to the simple linear processing required by the orthogonally designed STBC systems over flat-fading channels. In this section, we first analyze the asymptotic behavior of the system. The asymptotic study enlightens a new approach to designing a space-time equalizer under the constraint of linear processing at the receiver, which we shall discuss next.

From the analysis in the previous section, we know that the resulting bit error probability using the Alamouti algorithm can be expressed as $P_{b}=Q\left(\sqrt{\frac{2 E_{b} \gamma^{0}}{P_{00}^{1}+P_{10}^{1}+P_{00}^{2}+P_{10}^{2}+N_{0}}}\right)=Q\left(\sqrt{\frac{2 E_{b} \gamma^{0}}{N_{e}}}\right)$, where $N_{e}$ denotes the effective noise power. Apparently, there are two factors that contribute to its high suboptimality for the BFWA channels. First, the multipath diversity is not exploited, only the desired signal components from the first path of the two channels are combined $\left(\gamma_{0}=\left|h_{00}^{0}\right|^{2}+\left|h_{10}^{0}\right|^{2}\right)$. On the other hand, the noise component is greatly enlarged due to the ISI. Consequently, the effective noise power is far greater than the original noise power $\left(N_{e}=P_{00}^{1}+P_{10}^{1}+P_{00}^{2}+P_{10}^{2}+N_{0} \gg N_{0}\right)$. The $Q$-function is a monotonically decreasing function, meaning that in order to minimize the bit error probability $P_{b}$, we should maximize the numerator (the effective signal energy) and in the meantime minimize the denominator (the effective noise power). The best performance is obtained when the full multipath diversity is achieved so that $\gamma^{0}$ is replaced by $\gamma=\sum_{i, j, l}\left|h_{i j}^{l}\right|^{2}$, which is the total received power from different channels' different paths. The summation is carried out over all possible values of $i \in\{0,1\}$,

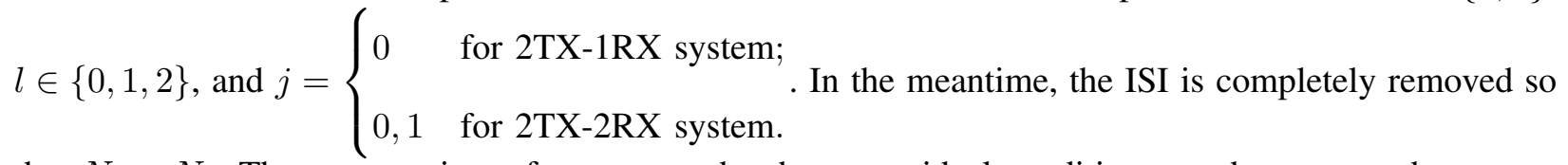
that $N_{e}=N_{0}$. The asymptotic performance under these two ideal conditions can be expressed as

$$
P_{b}^{\min }=Q\left(\sqrt{\frac{2 E_{b} \gamma}{N_{0}}}\right) .
$$

Next, we introduce some schemes in an attempt to enable the system to approach its theoretical performance limit expressed by (24).

\section{A. Diversity combining}

The first condition for the system to achieve the system's asymptotic performance expressed by (24) can be met by using a diversity combining technique. According to (15), the received signals at antenna 
rx0 are

$$
\begin{aligned}
r_{n}^{0} & =h_{00}^{2} s_{n-1}^{0}-h_{00}^{1} s_{n-1}^{1 *}+\underline{h_{00}^{0} s_{n}^{0}}+\ldots+w_{n}^{0} ; \\
r_{n}^{1} & =-h_{00}^{2} s_{n-1}^{1 *}+\underline{\underline{h_{00}^{1} s_{n}^{0}}}+\ldots+h_{10}^{1} s_{n}^{1}+\underline{h_{10}^{0} s_{n}^{0 *}}+w_{n}^{1} ; \\
r_{n+1}^{0} & =\underline{\underline{\underline{h_{00}^{2} s_{n}^{0}}}}-h_{00}^{1} s_{n}^{1 *}+\ldots+\underline{\underline{h_{10}^{1} s_{n}^{0 *}}}+h_{10}^{0} s_{n+1}^{1}+w_{n+1}^{0} ; \\
r_{n+1}^{1} & =-h_{00}^{2} s_{n}^{1 *}+\ldots+\underline{\underline{\underline{h_{10}^{2} s_{n}^{0 *}}}}+h_{10}^{1} s_{n+1}^{1}+\ldots+w_{n+1}^{1} .
\end{aligned}
$$

One can see from these equations that the desired symbols $s_{n}^{0}, s_{n}^{1}$ not only appear in the first-tap terms (with one line underneath), but also appear in the second-tap terms (with two lines underneath), as well as in the third-tap terms (with three lines underneath). Statistically, the first tap is the strongest tap in the SUI-3 channel (meaning $\mathrm{E}\left[\left|h_{i j}^{0}\right|^{2}\right]>\mathrm{E}\left[\left|h_{i j}^{1}\right|^{2}\right]$ and $\mathrm{E}\left[\left|h_{i j}^{0}\right|^{2}\right]>\mathrm{E}\left[\left|h_{i j}^{2}\right|^{2}\right]$ ). However, it is a quasi-static channel, channel coefficients change from block to block. For some channel realizations, the other taps can be stronger than the first one. Therefore, instead of always choosing the first tap as shown in (16), the Alamouti scheme can be applied on the strongest tap based on the channel statistic for a specific channel realization. Furthermore, in order to take advantage of multipath propagation and obtain diversity gain, we should apply the Alamouti scheme on the two strongest or all the three taps and combine the desired signals from different taps. The modified Alamouti algorithm based on three-path combining can be expressed as

$$
\begin{aligned}
& \widetilde{s_{n}^{0}}=\hat{h}_{00}^{0 *} r_{n}^{0}+\hat{h}_{10}^{0} r_{n}^{1 *}+\hat{h}_{00}^{1 *} r_{n}^{1}+\hat{h}_{10}^{1} r_{n+1}^{0 *}+\hat{h}_{00}^{2 *} r_{n+1}^{0}+\hat{h}_{10}^{2} r_{n+1}^{1 *}=\sum_{i, l} \hat{h}_{i 0}^{l *} h_{i 0}^{l} s_{n}^{0}+\epsilon_{n}^{0} \approx \gamma s_{n}^{0}+\epsilon_{n}^{0} ; \\
& \widetilde{s_{n}^{1}}=\hat{h}_{10}^{0 *} r_{n}^{0}-\hat{h}_{00}^{0} r_{n}^{1 *}+\hat{h}_{10}^{1 *} r_{n}^{1}-\hat{h}_{00}^{1} r_{n+1}^{0 *}+\hat{h}_{10}^{2 *} r_{n+1}^{0}-\hat{h}_{00}^{2} r_{n+1}^{1 *}=\sum_{i, l} \hat{h}_{i 0}^{l *} h_{i 0}^{l} s_{n}^{1}+\epsilon_{n}^{1} \approx \gamma s_{n}^{1}+\epsilon_{n}^{1},
\end{aligned}
$$

where $\hat{h}_{i 0}^{l}$ is an estimate of $h_{i 0}^{l}$, and $\gamma=\sum_{i, l}\left|h_{i 0}^{l}\right|^{2}$ is the total received power from different paths, and $\epsilon_{n}^{0}, \epsilon_{n}^{1}$ are the combined noise and ISI terms.

\section{B. Interference cancellation (IC)}

The summation in (26) is carried out over all possible values of $i \in\{0,1\}$, and $l \in\{0,1,2\}$. One can see that this combining scheme also leads to temporal diversity gain in addition to the spatial diversity gain obtained by the original Alamouti scheme. The first condition for achieving the system's asymptotic performance is approached (or fully fulfilled given perfect channel estimation) by applying the multipath combining scheme introduced above. On the other hand, however, $\epsilon_{n}^{0}, \epsilon_{n}^{1}$ in (26) contain more ISI terms compared to $w_{n}^{0}, w_{n}^{1}$ in (16), which in turn will have a detrimental effect on the overall system performance. In order to tackle this problem, we employ the multistage interference cancellation technique, in an attempt to cancel the contribution of the ISI and achieve the ISI-free performance expressed by (24).

Let us denote $\hat{s}_{n-i}^{0}, \hat{s}_{n-i}^{1}$ as an estimate of $s_{n-i}^{0}, s_{n-i}^{1}$ from the previous stage. To simplify the notation, the iteration (stage) index is omitted whenever no ambiguity arises. Given a channel estimate $\hat{h}_{i j}^{l}$ and 
symbol estimates $\left\{\hat{s}_{n-i}^{0}, \hat{s}_{n-i}^{1}\right\}$, the ISI canceled version of the received signal $r_{n}^{0}$, denoted as $\bar{r}_{n}^{0}$ can be written according to (15) as

$$
\begin{aligned}
\bar{r}_{n}^{0} & =\left(h_{00}^{2} s_{n-1}^{0}-\hat{h}_{00}^{2} \hat{s}_{n-1}^{0}\right)-\left(h_{00}^{1} s_{n-1}^{1 *}-\hat{h}_{00}^{1} \hat{s}_{n-1}^{1 *}\right)+h_{00}^{0} s_{n}^{0} \\
& +\left(h_{10}^{2} s_{n-1}^{1}-\hat{h}_{10}^{2} \hat{s}_{n-1}^{1}\right)+\left(h_{10}^{1} s_{n-1}^{0 *}-\hat{h}_{10}^{1} \hat{s}_{n-1}^{0 *}\right)+h_{10}^{0} s_{n}^{1}+w_{n}^{0} .
\end{aligned}
$$

Other ISI canceled versions of the received signals, e.g., $\bar{r}_{n}^{1}, \bar{r}_{n+1}^{0}, \bar{r}_{n+1}^{1}$ can be formed similarly, i.e., by canceling the contribution from the symbols other than $s_{n}^{0}, s_{n}^{1}$. Using the aforementioned combining technique, the soft decisions of $s_{n}^{0}, s_{n}^{1}$ can now be formed based upon the ISI canceled signals as

$$
\begin{aligned}
& \widetilde{s_{n}^{0}}=\hat{h}_{00}^{0 *} \bar{r}_{n}^{0}+\hat{h}_{10}^{0} \bar{r}_{n}^{1 *}+\hat{h}_{00}^{1 *} \bar{r}_{n}^{1}+\hat{h}_{10}^{1} \bar{r}_{n+1}^{0 *}+\hat{h}_{00}^{2 *} \bar{r}_{n+1}^{0}+\hat{h}_{10}^{2} \bar{r}_{n+1}^{1 *}=\sum_{i, l} \hat{h}_{i 0}^{l *} h_{i 0}^{l} s_{n}^{0}+\varepsilon_{n}^{0} \approx \gamma s_{n}^{0}+\varepsilon_{n}^{0} ; \\
& \widetilde{s_{n}^{1}}=\ldots=\sum_{i, l} \hat{h}_{i 0}^{l *} h_{i 0}^{l} s_{n}^{1}+\varepsilon_{n}^{1} \approx \gamma s_{n}^{1}+\varepsilon_{n}^{1},
\end{aligned}
$$

where $\varepsilon_{n}^{0}, \varepsilon_{n}^{1}$ denote the noise plus cancellation residual. Given correct decision feedback, all the ISI terms will be eliminated. The variance of $\varepsilon_{n}^{0}, \varepsilon_{n}^{1}$ will be much smaller than that of $\epsilon_{n}^{0} \epsilon_{n}^{1}$ in (26) and $w_{n}^{0} w_{n}^{1}$ in (16), consequently, the BER performance will be greatly improved. From (27) and (28), we see that this scheme does not involve any matrix inversion, it only requires linear processing at the receiver.

\section{Study of asymptotic performance}

With the aim of combatting ISI and exploiting temporal diversity, a space-time equalization scheme is developed based on the IC and multipath combining techniques in Section IV-A and IV-B. We shall now analyze the performance bound of this equalization scheme in order to gain an insight into its asymptotic performance. It is studied based on the assumption that the interference cancellation and channel estimation are perfect. That would be the ideal situation leading to best achievable performance. The condition of perfect interference cancellation can be approached by proper design of equalization and decoding so that ISI-free transmission can be acheived for some frequency selective fading channels [16]. It was shown in [17] that the error due to imperfect channel estimation can be made arbitrarily small given sufficient pilot symbols. Given perfect channel estimation, i.e., $\hat{h}_{i 0}^{l *}=h_{i 0}^{l *}$, then $\sum_{i, l} \hat{h}_{i 0}^{l *} h_{i 0}^{l}=\gamma$. Equation (28) holds with equality, i.e., $\widetilde{s_{n}^{0}}=\gamma s_{n}^{0}+\varepsilon_{n}^{0}$. Given perfect interference cancellation, all the ISI terms in (28) will be eliminated. In this case,

$$
\varepsilon_{n}^{0}=h_{00}^{0 *} w_{n}^{0}+h_{10}^{0} w_{n}^{1 *}+h_{00}^{1 *} w_{n}^{1}+h_{10}^{1} w_{n+1}^{0 *}+h_{00}^{2 *} w_{n+1}^{0}+h_{10}^{2} w_{n+1}^{1 *}
$$

Its variance can be easily derived as $N_{\varepsilon}=\sum_{i, l}\left|h_{i 0}^{l}\right|^{2} N_{0}=\gamma N_{0}$, using the fact that the taps with different delays are uncorrelated within a channel as well as between channels (e.g., $h_{10}^{0}$ is uncorrelated with $h_{00}^{1}$, and $h_{10}^{1}$ is uncorrelated with $h_{00}^{2}$ ). Following the same procedure as in Section III, the performance obtained under the assumption of perfect channel estimation and cancellation can be derived as $P_{b}(\gamma)=Q\left(\frac{A \gamma}{\sqrt{N_{\varepsilon} / 2}}\right)=Q\left(\sqrt{\frac{2 E_{b} \gamma}{N_{0}}}\right)$, which is the exactly same as the asymptotic performance 
expressed by (24). We therefore come to the conclusion that the theoretical limit of the system performance can be reached with the diversity combining and the IC techniques under the condition of perfect channel estimation and perfect cancellation. In order to compute the average asymptotic bit error probability, let us form a channel vector $\mathbf{h}=\left[\begin{array}{llllll}h_{00}^{0} & h_{10}^{0} & h_{00}^{1} & h_{10}^{1} & h_{00}^{2} & h_{10}^{2}\end{array}\right]^{T}$. The joint PDF of $\mathbf{h}$ is determined by its mean vector $\mathbf{m}_{\mathbf{h}}$ and its covariance matrix $\mathbf{C}_{\mathbf{h}}=\mathrm{E}\left\{\left[\mathbf{h}-\mathbf{m}_{\mathbf{h}}\right]\left[\mathbf{h}-\mathbf{m}_{\mathbf{h}}\right]^{*}\right\}$, i.e.,

$$
p(\mathbf{h})=\frac{1}{(2 \pi)^{2}\left(\operatorname{det} \mathbf{C}_{\mathbf{h}}\right)^{1 / 2}} \exp \left[-\frac{1}{2}\left(\mathbf{h}-\mathbf{m}_{\mathbf{h}}\right)^{*} \mathbf{C}_{\mathbf{h}}^{-1}\left(\mathbf{h}-\mathbf{m}_{\mathbf{h}}\right)\right]
$$

Since $\gamma=\mathbf{h}^{*} \mathbf{h}$, and $P_{b}(\mathbf{h})=Q\left[\sqrt{\frac{2 E_{b} \mathbf{h}^{*} \mathbf{h}}{N_{0}}}\right]$, the average bit error probabilities can be formed as

$$
\begin{aligned}
\bar{P}_{b} & =\int_{\mathbf{h}} P_{b}(\mathbf{h}) p(\mathbf{h}) d \mathbf{h}=\int_{\mathbf{h}} Q\left[\sqrt{\frac{2 E_{b} \mathbf{h}^{*} \mathbf{h}}{N_{0}}}\right] p(\mathbf{h}) d \mathbf{h} \\
& =\frac{1}{\pi} \int_{0}^{\frac{\pi}{2}}\left[\operatorname{det}\left(\frac{\boldsymbol{\Sigma}}{\sin ^{2} \theta}+\mathbf{I}\right)\right]^{-1} \exp \left[-\mathbf{m}^{*}\left(\boldsymbol{\Sigma}+\sin ^{2} \theta \mathbf{I}\right)^{-1} \mathbf{m}\right] d \theta,
\end{aligned}
$$

where $\mathbf{m}=\mathbf{m}_{h} / \beta, \boldsymbol{\Sigma}=\mathbf{C}_{\mathbf{h}} / \beta^{2}, \beta=\sqrt{N_{0} / E_{b}}$. Equation (31) is the performance bound for the 3-path combining scheme. The asymptotic performance of the other schemes can be derived similarly.

\section{Analytical Results And Performance Comparison}

A comparison between analytical and simulation results is presented in this section in order to verify the theoretical analysis conducted in the previous sections. The simulation curves are obtained numerically by averaging the results over 1000 channel realizations. During each Monte-Carlo run, the block size is set to 10000 bits, which corresponds to 5000 QPSK symbols. The channel coefficients vary from one block to another, however, they are assumed to remain constant during the transmission of one block of data. In the simulations, we assume perfect knowledge of the channel state information (CSI). The noise variance $N_{0}$ and path delays are assumed to be known to the receiver. For the SUI-3 SISO channel, the total channel gain $\sum_{l=0}^{2} \mathrm{E}\left[\left|h_{l}\right|^{2}\right]=\sum_{l=0}^{2} P_{l}$ is normalized to unity, and the parameters are set accordingly to $\sigma^{2}=0.175, s^{2}=0.36, P_{1}=0.223, P_{2}=0.07$ in equation (9), (14). For the MIMO channels, the antenna correlation coefficient defined in (19) is set to 0.4 unless otherwise stated. The channel statistics are summarized as follows: $E\left[h_{00}^{0}\right]=E\left[h_{10}^{0}\right]=0.4243+0 j ; \quad E\left[h_{00}^{1}\right]=E\left[h_{00}^{2}\right]=E\left[h_{10}^{1}\right]=E\left[h_{10}^{2}\right]=0+$ $0 j ; \quad P_{00}^{0}=P_{10}^{0}=E\left[\left|h_{00}^{0}\right|^{2}\right]=E\left[\left|h_{10}^{0}\right|^{2}\right]=0.3536 ; \quad P_{00}^{1}=P_{10}^{1}=E\left[\left|h_{00}^{1}\right|^{2}\right]=E\left[\left|h_{10}^{1}\right|^{2}\right]=\operatorname{var}\left[h_{00}^{1}\right]=$ $\operatorname{var}\left[h_{10}^{1}\right]=0.1115 ; \quad P_{00}^{2}=P_{10}^{2}=E\left[\left|h_{00}^{2}\right|^{2}\right]=E\left[\left|h_{10}^{2}\right|^{2}\right]=\operatorname{var}\left[h_{00}^{2}\right]=\operatorname{var}\left[h_{10}^{2}\right]=0.035 ; \quad \operatorname{var}\left[h_{00}^{0}\right]=$ $\operatorname{var}\left[h_{10}^{0}\right]=E\left[\left|h_{00}^{0}\right|^{2}\right]-\left|E\left[h_{00}^{0}\right]\right|^{2}=0.174 ; \quad P_{00}^{0}+P_{00}^{1}+P_{00}^{2}+P_{10}^{0}+P_{10}^{1}+P_{10}^{2}=1.0$.

Fig. 2 shows that the results of the theoretical analysis for the coherent QPSK system expressed by equation (14) are in close agreement with the simulation results. The truncation value $N=10$ in (14) is noticed to be sufficient. All the curves lie above $B E R=10^{-1}$, which is far from satisfactory. Clearly, coherent detection alone cannot cope with the effect of ISI which is inherent in the multipath SUI-3 BFWA channels. 
Fig. 2 also shows that the analysis and simulation results match quite closely for both $2 \mathrm{TX}-1 \mathrm{RX}$ and 2TX-2RX systems, although the discrepancy is larger in the latter case. Comparing the SISO system with the MIMO systems, one can see that employing multiple antennas improves the system performance, especially with $2 \mathrm{TX}-2 \mathrm{RX}$ antennas. However, the improvement is still quite limited when the original Alamouti algorithm is applied. As in the SISO case, an irreducible error floor is observed even with $2 \mathrm{TX}-2 \mathrm{RX}$ antennas. The bit error rate cannot be further reduced by increasing $E_{b} / N_{0}$. The reason is simply that the Alamouti algorithm was developed for flat fading channels and so does not take into consideration the ISI introduced by the SUI-3 BFWA channel. For the BFWA channels, which exhibit frequency-selectivity as the transmission rate increases, equalization becomes indispensable.

Next, we present the results for the space-time equalization algorithms introduced in Section IV-A and IV-B. It is based on the Alamouti algorithm, which has been modified in such way that both spatial and temporal diversities are exploited and the effect of ISI is mitigated. The basic idea is to apply multistage interference cancellation technique to cancel the contribution of the ISI before applying the Alamouti detection scheme. In addition to the direct implementation of the Alamouti detection on the ISI canceled signals (1-path non-selective), we can also apply the Alamouti detection on the strongest or the two strongest or all the three taps and combine desired signals from different taps in order to take advantage of multipath propagation and obtain diversity gain. We call them 1-path selective, 2path and 3-path scheme, respectively. In our simulations, the number of cancellation stages is set to 6 , since it is observed that all the algorithms would converge after 6 stages. At the initial stage, we use the 3-path combining scheme (see (26)) to get a crude symbol estimate. At the following stages, the modified Alamouti algorithm with IC (see (27) and (28)) is employed to cancel ISI using decision feedback from the previous stage. Fig. 3 shows the performance of the proposed algorithms for the $2 \mathrm{TX}-1 \mathrm{RX}$ and $2 \mathrm{TX}-2 \mathrm{RX}$ systems, respectively. In both cases, the IC plus 1-path non-selective scheme has the worst performance. However, it is somewhat surprising that the 2-path and 3-path schemes yield higher BER than the 1-path selective scheme. The rationale is that the IC scheme is prone to the error propagation problem. Cancellation using incorrect decision will increase interference rather than canceling interference. Decision feedback errors have more detrimental effects on the 2(3)-path schemes since they contain more cancellation residual terms.

The asymptotic performance of the proposed schemes are demonstrated in Fig. 4. The analytical bound is given by (31); the simulated bound is obtained by assuming perfect knowledge of the transmitted symbols and CSI in the simulations so that the cancellation is perfect. The plot shows fairly close agreement between the analysis and simulations, especially for the 1-path and 2-path schemes. This means that the derived theoretical bounds are tight bounds, they provide good insights into the asymptotic behavior of the proposed algorithms. Fig. 4 shows that the error floor does not appear for the performance bounds. It also indicates that the IC plus 3-path combining scheme achieves full temporal diversity. Once the error propagation problem is tackled, it will outperform all the other schemes. By comparing Fig. 4 
with Fig. 3.a), it is obvious that the diversity combining and IC schemes are far beyond their performance bounds. The errors in the decision feedback significantly degrade performance and prevent the algorithms from reaching their theoretical potential. The solution will be addressed shortly in next section.

All the results demonstrated above are obtained assuming $\rho=0.4$. The impact of the spatial correlation is examined in Fig. 5 using the analytical performance bound of the 3-path scheme as an example. Clearly, antenna correlation has a detrimental effect on the system performance. The higher the correlation coefficient, the worse performance one can expect. Consequently, it is desirable to reduce the correlations in the practical MIMO systems, for example, by increasing the antenna spacings at the transmitter and at the receiver.

\section{TURBO EQUALIZATION ALGORITHM}

We see from the above results that the proposed equalization scheme is far from its asymptotic performance. The errors in the decision feedback cause significant performance degradation and prevent the algorithm from reaching its theoretical limit. There are various ways of tackling this problem, e.g., introducing channel coding to reduce the feedback error probability, and using soft cancellation rather than brute force cancellation to prevent error propagation. These ideas combined with turbo processing principle [18] lead to a new approach to space-time turbo equalization which will be described next.

In order to reduce the error propagation and exploit the potential offered by the previously described equalization algorithm, we apply a convolutional code to the system. The information sequence $\left\{b_{n}\right\}$ is convolutionally encoded into coded bits $\left\{u_{n}\right\}$, which are subsequently interleaved and each block of two coded and interleaved bits $u_{n}^{\prime}[0], u_{n}^{\prime}[1]$ is mapped into one of the four QPSK symbols. The QPSK symbols are space-time coded according to Alamouti scheme and transmitted over the BFWA channel. The received signal is basically the same as (15) except that the QPSK symbols are formed by coded bits rather than information bits.

The proposed space-time turbo equalization algorithm is based on the turbo processing principle, which was first employed for decoding parallel concatenated convolutional codes, known as turbo codes [19]. With turbo processing, each subsystem is implemented with a soft-input, soft-output algorithm. Soft decision values, typically in the form of log-likelihood ratios (LLRs), are passed down the chain and refined by the subsequent stages. The soft output of the final stage is then fed back to the first stage and a second iteration of the processing is initiated. Several iterations of turbo processing can be executed to improve performance. The proposed turbo equalization algorithm is illustrated in Fig. 6. First, we use a training sequence to acquire a channel estimate $\hat{h}_{i j}^{l}$ using some channel estimation algorithm. In the meantime, a modified Alamouti algorithm is used to obtain the soft values of the transmitted symbols in the form of log-likelihood ratio (LLR) $\left\{\lambda\left(s_{n}\right)=\lambda\left(x_{n}\right)+j \lambda\left(y_{n}\right)\right\}$ where $s_{n}$ denotes either $s_{n}^{0}$ or $s_{n}^{1}$.

The channel estimate $\hat{h}_{i j}^{l}$ and symbol estimates $\left\{\lambda\left(s_{n}\right)\right\}$ are passed to the equalizer, which computes $\widetilde{s_{n}}$, the soft decision of $s_{n}$ (the equalizer is designed based on the schemes described in Section IV-A 
and IV-B). The soft estimate of the symbol is then mapped to the LLR values of coded bits $\left\{\lambda\left(u_{n}^{\prime} ; O\right)\right\}$ by the symbol-to-bit converter (SBC), which are deinterleaved to yield $\left\{\lambda\left(u_{n} ; I\right)\right\}$. The interleaver and deinterleaver are denoted as $\Pi$ and $\Pi^{-1}$ in Fig. 6. Based on the soft inputs, a Log-MAP decoder computes the LLR for each information bit $\lambda\left(b_{n} ; O\right)$ and each coded bit $\lambda\left(u_{n} ; O\right)$. The former is used to make decisions on the transmitted information bit at the final iteration, and the latter is interleaved and passed through a bit-to-symbol converter (BSC) to derive a soft symbol estimate $\lambda\left(s_{n}\right)$, which is used for equalization at the next iteration. We use the notations $\lambda(\cdot ; I)$ and $\lambda(\cdot ; O)$ to denote the input and output ports of a soft-input and soft-output device.

Numerical results are presented next to assess the performance of the proposed turbo equalization scheme. We employ a rate 1/3 Maximum Free Distance convolutional code with constraint length 5 and generator polynomials $(25,33,37)$ in octal form. During each Monte-Carlo run, the block size is set to 2000 information bits followed by 4 tails bits to terminate the trellis, which corresponds to $2004 \times 3=6012$ coded bits or 3006 QPSK symbols, 200 of which are used as pilots to acquire a channel estimate $\hat{h}_{i j}^{l}$. Channel estimation is conducted with the modified maximum likelihood algorithm introduced in [17]. The coded bits are interleaved by a random interleaver. The simulation curves are obtained by averaging the simulation results over at least 300 channel realizations. In order to ensure the reliability of the performance measurements at low error rate, a sufficient number of data blocks are transmitted so that at least 100 errors are generated. To study the behavior of each algorithm, the number of stages is set to 4 since it is observed that no more than 4 stages (in addition to the non-cancellation stage) are needed for the discussed schemes to converge.

Different combining (selection) strategies are compared in Fig. 7 for the 2TX-1RX and 2TX-2RX STBC BFWA systems. The 1-path schemes yield the worst results. We also see that the 1-path selective scheme performs better than the 1-path non-selective one, the 2-path scheme is superior to the 1-path schemes, and the the 3-path scheme is the best of all. The results concur with our study of the asymptotic behavior for the space-time equalization scheme demonstrated in Fig. 4. Obviously, in order to fully achieve the temporal diversity from the multipath propagation, we need to combine the signals from all the paths. As illustrated in Fig. 7, the error propagation problem is effectively solved, and the error floor presented in the uncoded system is eliminated. The potential for performance improvement predicted in Fig. 4 is realized by extending the algorithm to the coded systems and by applying the turbo processing principle.

As demonstrated in Fig. 7, the performance gain by adding one receiver antenna is approximately 5 $\mathrm{dB}^{1}$. By comparing Fig. 7 with Fig. 3, we learned that the advantages of using multiple antennas are more significant for the coded system than for the uncoded system, especially when turbo equalization is used. The 3-tap turbo equalizer is also investigated using the perfect channel state information (CSI)

\footnotetext{
${ }^{1}$ Here $E_{b}$ refers to the transmitted bit energy, and is not affected by the number of receiver antennas. The gain would be $2 \mathrm{~dB}$ if we define $E_{b}$ as the received bit energy.
} 
and the result is shown in Fig. 7.b) for the 2TX-2RX STBC BFWA system. Compared to the results obtained using channel estimation, the performance loss due to the channel estimation errors is within a fraction of $1 \mathrm{~dB}$.

In Fig. 8, the proposed scheme is compared with the linear MMSE filter based turbo equalization and time-reversal STBC (TR-STBC). The proposed scheme performs much better than the MMSE based turbo equalization due to the reason that the proposed scheme not only preserves the features of the original Alamouti algorithm, i.e., achieving spatial diversity with simple linear processing at the receiver, but also takes advantage of the multipath propagation and obtains the temporal diversity by multipath combining and reduces the effect of ISI by interference cancellation. On the contrary, direct implementation of the MMSE algorithm on the STBC coded systems cannot utilize the spatial diversity as discussed in [20].

The TR-STBC is a transmit diversity scheme specially designed for frequency selective channels [21]. It converts MIMO multipath channel to several parallel SISO multipath channels, leading to significant simplification of receiver design. The TR-STBC itself only decouples the symbol streams from two transmit antennas. It, however, does not resolve the ISI in each symbol stream. The ISI of course still has to be handled by an equalizer. To this end, we can use some linear equalizer, e.g., ZF or MMSE equalizer to mitigate the effect of the ISI. The MMSE equalizer is usually preferred since the ZF equalizer causes noise enhancement. Therefore, we apply a 7-tap MMSE equalizer in the TR-STBC system after the symbol streams are decoupled. As shown in Fig. 8, the proposed turbo equalization scheme outperforms the TR-STBC scheme with MMSE equalizer by over $1 \mathrm{~dB}$. Equalizers with length greater than 7 are tested for the TR-STBC system and are shown to have very marginal gain compared to 7-tap equalizer. Note that the proposed scheme has a higher computational complexity than the TR-STBC plus ZF/MMSE equalization, and the overhead is mainly imposed by the turbo processing procedure which involves the space-time equalization and decoding in an iterative manner.

In addition to the single-carrier modulation with time-domain equalization discussed previously, there are some other alternatives to combat the effect of ISI, e.g., using orthogonal frequency division multiplexing (OFDM) or single-carrier modulation with frequency-domain equalization (SC-FDE). Compared to the OFDM solution, SC-FDE is often preferred since it achieves similar performance and complexity to that of OFDM, while at the same time, being less sensitive to transmitter nonlinearities and phase noise [24][26]. A detailed description of the SC-FDE scheme for STBC coded transmission over frequency-selective channels was presented in [24]; and the application of the SC-FDE scheme to SISO and MIMO BFWA systems was given in [25] and [26], respectively. By comparing the results shown in this paper with those presented in [26], we can conclude that the proposed scheme yields superior performance to the SCFDE technique for MIMO BFWA channels. However, the proposed scheme has a higher computational complexity for the reason stated previously.

The results presented in this paper are relevant to a generic single carrier based BFWA system, and not to IEEE 802.16 specifically. In particular, only a single convolutional coding scheme is investigated, 
however, it should be noted that in the IEEE 802.16 standard, the inner convolutional code is concatenated with an outer Reed-Solomon code over GF(256) with variable block size and error correction capabilities to robustly transmit critical data, such as frame control and initial accesses. Some optional coding schemes are also specified in the standard, such as a convolutional turbo code (CTC) and a block turbo code (BTC). Note that a comparative study on the performance of convolutional codes and turbo codes for BFWA systems has been conducted in [27]. Compared to the convolutional codes with the same decoder complexity, turbo codes have similar performance in SISO BFWA channel, and achieve better performance in MIMO BFWA channel as the number of antennas increases [27]. When the inner convolutional/turbo code is concatenated with an outer Reed-Solomon code, the system performance will improve at the cost of increased encoding and decoding complexity. How to design an iterative detection scheme using turbo processing principle for a MIMO BFWA system with serially concatenated convolutional code and Reed-Solomon code is a future research topic for the authors.

\section{CONCLUSIONS}

In this paper, we first analyze the performance of conventional coherent detection and Alamouti detection for SISO and MIMO BFWA systems. Comparison with simulation results shows that the analysis is reasonably accurate. Both simulation and analysis indicate that the SUI-3 BFWA channel is very hostile, consequently, conventional detection schemes will therefore not suffice. The performance improvement by applying multiple antennas is quite limited owing to the presence of ISI and antenna correlation. Asymptotic performance of the BFWA MIMO system is then investigated, and our theoretical study indicates that the temporal diversity has to be exploited and the effect of ISI has to be removed in order to approach the theoretical capacity of the system. The temporal diversity can be obtained by multipath combining, and the effect of ISI reduced by interference cancellation. The results show that the error propagation problem causes an irreducible error floor and prevents the system from reaching its theoretical limit. To tackle this problem, we extend the algorithm to a convolutionally coded STBC system and apply turbo processing principle, resulting in a space-time turbo equalization algorithm which is shown to have superior performance to the conventional linear MMSE filter based turbo equalization and the TR-STBC transmit diversity scheme for BFWA channels. In addition, it only requires linear processing at the receiver, which makes it a feasible solution for practical implementation.

\section{ACKNOWLEDGMENT}

This work is sponsored by EPSRC under Grant GR/S46444. The authors would like to acknowledge its financial support. 


\section{REFERENCES}

[1] IEEE 802.16 Working Group on Broadband Wireless Access Standards. available at http://grouperieee.org/groups/802/16/. 2002.

[2] Draft ETSI TS 101, Broadband Radio Access Networks (BRAN), HIPERMAN. available at http://portal.etsi.org/bran/Summary.asp, 2002.

[3] C. Eklund. "IEEE Standard 802.16: a technical overview of the wirelessMAN air interface for broadband wireless access". IEEE Communications Magazine, pp. 98-107, June, 2002.

[4] H. Bolcskei, A. Paulraj, K. Hari, R. Nabar, W. Lu. "Fixed broadband wireless access: state of the art, challenges, and future directions". IEEE Communication Magazine, pp. 100-108, Jan. 2001.

[5] W. Webb. "Broadband fixed wireless access as a key component of the future integrated communications environment". IEEE Communications Magazine, pp. 115-121, Sept. 2001.

[6] V. Erceg. "An empirically based path loss model for wireless channels in suburban environments". IEEE JSAC, vol. 17, no. 7, pp. 1205-1211, July 1999.

[7] J. Proakis. Digital Communications, 3rd edition, McGraw-Hill, 1995.

[8] S. Verdu. Multiuser Detection, 1st edition, Cambridge University Press, 1998.

[9] V. Tarokh, N. Seshadri, A. Calderbank. "Space-time codes for high data rates wireless communications: performance criterion and code construction”. IEEE Transactions on Information Theory, vol. 44, pp. 744-765, March 1998.

[10] A. Alamouti. “A simple transmit diversity technqiue for wireless communications". IEEE Journal on Selected Areas in Communications, vol. 16, no. 8, pp. 1451-1458, Oct. 1998.

[11] V. Tarokh, N. Seshadri, A. Calderbank. "Space-time block coding from orthogonal designs". IEEE Transactions on Information Theory, vol. 45, pp. 1456-1467, July 1999.

[12] S. Sandhu, A. Paulraj. "Space-time block codes versus space-time trellis codes", Proceedings IEEE Internation Conference on Communications, vol. 4, pp. 1132-1136, 2001.

[13] M. Simon, M. Alouini. "A unified approach to the performance analysis of digital communication over generalized fading channels", Proceedings of the IEEE, vol. 86, no. 9, pp. 1860-1877, Sept. 1998.

[14] V. Veeravalli. "On performance analysis for signalling on correlated fading channels". IEEE Transactions on Communications, vol. 49, no. 11, pp. 1879-1883, Nov. 2001.

[15] L. Li, Y. Yao, H. Li "Channel estimation and equalization for space-time block coded systems in frequency selective fading channels”. IEEE Globecom'2001, vol. 1, pp. 300-304, Nov. 2001.

[16] C. Laot, A. Glavieux, Joel Labat. "Turbo equalization: adaptive equalization and channel decoding jointly optimized". IEEE Journal on Selected Areas in Communications, vol. 19, no. 9, Sept. 2001.

[17] P. Xiao, R. Carrasco, I. Wassell. "Estimation of FWA MIMO channels". Proc. IEEE Information Theory Workshop 2006. To appear.

[18] J. Hagenauer. "The Turbo principle: tutorial introduction and state of the art". Proc. International Symposium on Turbo Codes, pp. 1-11, Sept. 1997.

[19] C. Berrou, A. Glavieux, P. Thitimajshima. "Near Shannon limit error correcting coding and decoding: turbo-codes". Proc. IEEE International Conference on Communications, vol. 2, pp. 1064-1070, May 1993, Switzerland.

[20] P. Xiao, R. Carrasco, I. Wassell. "AML turbo equalization for space-time block coded systems". The Mediterranean Journal of Computers and Networks, Special Issue on Mobile Communication Theory, Technology and Applications. Accepted for publication.

[21] E. Lindskog, A. Paulraj. "A transmit diversity scheme for channels with intersymbol interference". IEEE International Conference on Communications, pp. 307-311, June 2002.

[22] J. Holtzman. "A simple, accurate method to calculate spread-spectrum multiple-access error probabilities". IEEE Transactions on Communications, vol. 40, no. 3, pp. 461-464, March 1992.

[23] Alan Jefferey. Handbook of Mathematical Formulas and Integrals, Academic Press, 2nd edition. 
[24] N. Al-Dhahir. "Single-carrier frequency-domain equalization for space-time block-coded transmission over frequencyselective channels". IEEE Communications Letters, vol. 5, no. 7, pp. 304-306, July 2001.

[25] D. Falconer, S. Ariyavisitakul, A. Benyamin-Seeyar, B. Eidson. "Frequency domain equalization for single-carrier broadband wireless systems”. IEEE Communications Magazine, vol. 40, no. 4, pp. 58-66, April 2002.

[26] I. Chatzigeorgiou, M. Rodrigues, I. Wassell, R. Carrasco. "Turbo coded OFDM/SC-FDE techniques for MIMO BFWA channels". Proc. International Symposium on Broadband Communications 2004, 2004, UK. Available at http://www.cl.cam.ac.uk/Research/DTG/ ic231/research/ISBC04_PrFriendly.pdf

[27] I. Chatzigeorgiou, M. Rodrigues, I. Wassell, R. Carrasco. "A comparison of convolutional and turbo coding schemes for broadband FWA systems". Proc. 12th International Conference on Telecommunications, May 2005, South Africa. Available at http://www.cl.cam.ac.uk/Research/DTG/publications/public/ic231/ICT05_CameraReady_Paper.pdf 


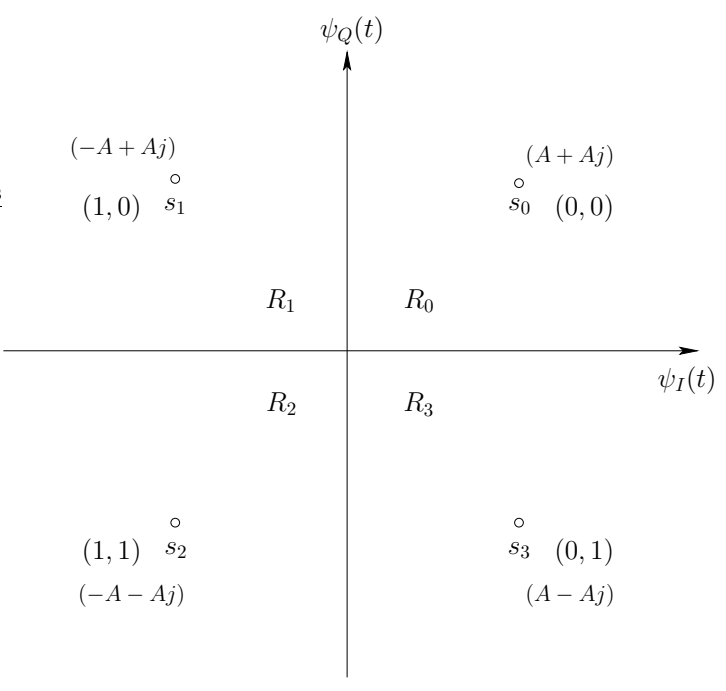

Fig. 1. QPSK constellation and decision regions. 


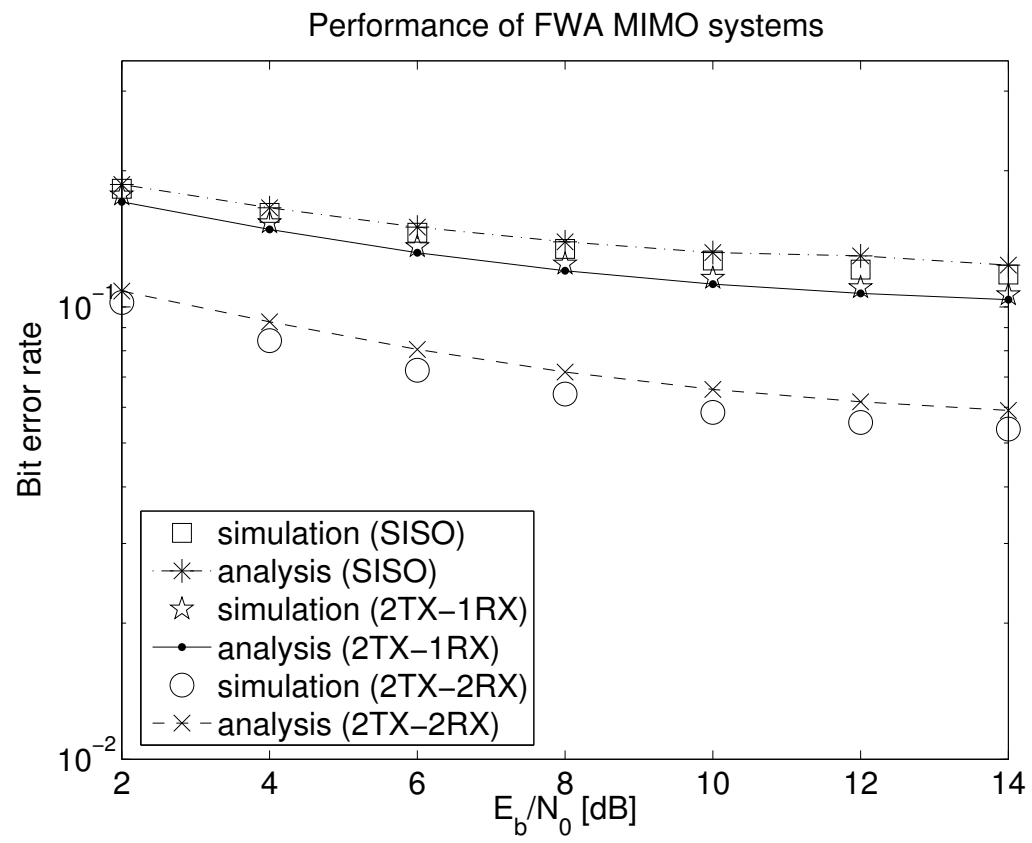

Fig. 2. Performance of coherent detection for SUI-3 SISO BFWA channel and the Alamouti scheme for SUI-3 BFWA multiple antenna channel. Antenna correlation $\rho=0.4$. 


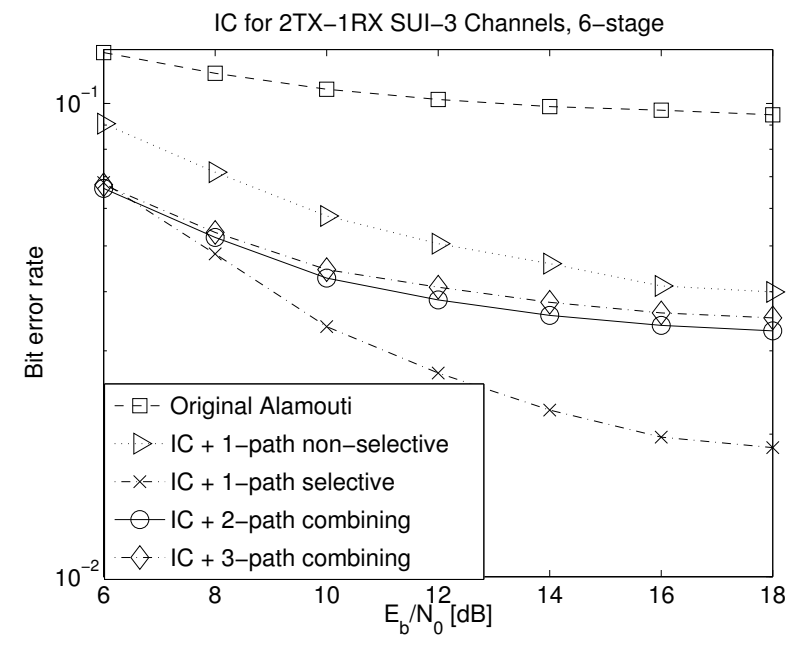

(a) $2 \mathrm{TX}-1 \mathrm{RX}$ System.

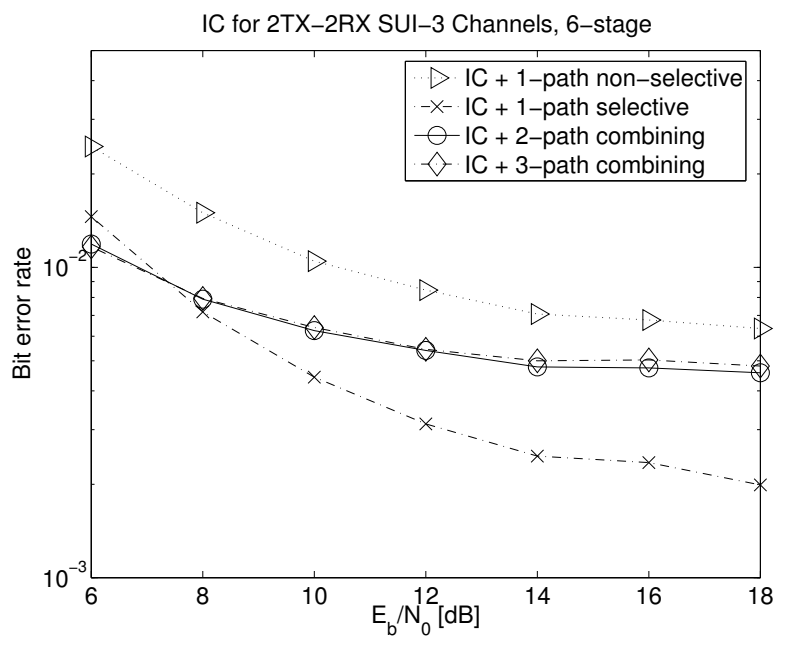

(b) 2TX-2RX System.

Fig. 3. Comparison of different schemes for the BFWA system. All the curves represent the 6th stage. 


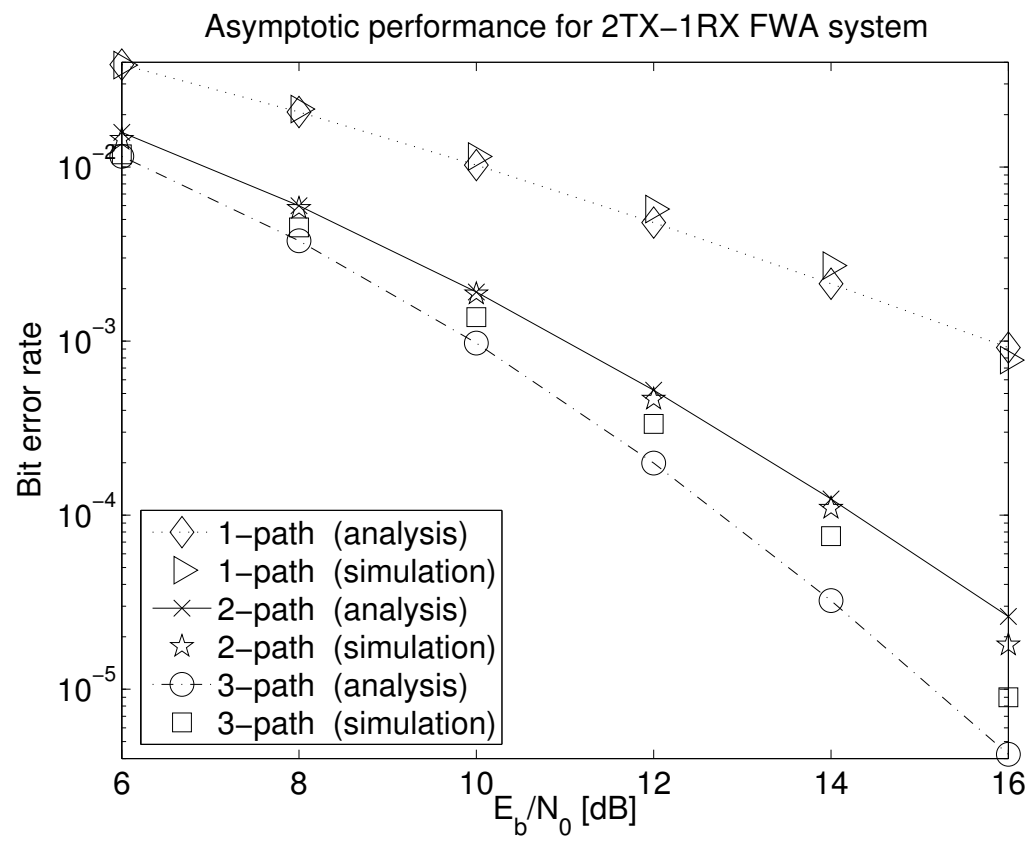

Fig. 4. Performance bounds of the proposed schemes for the 2TX-1RX system over SUI-3 channel (1-path scheme refers to the non-selective one here). 


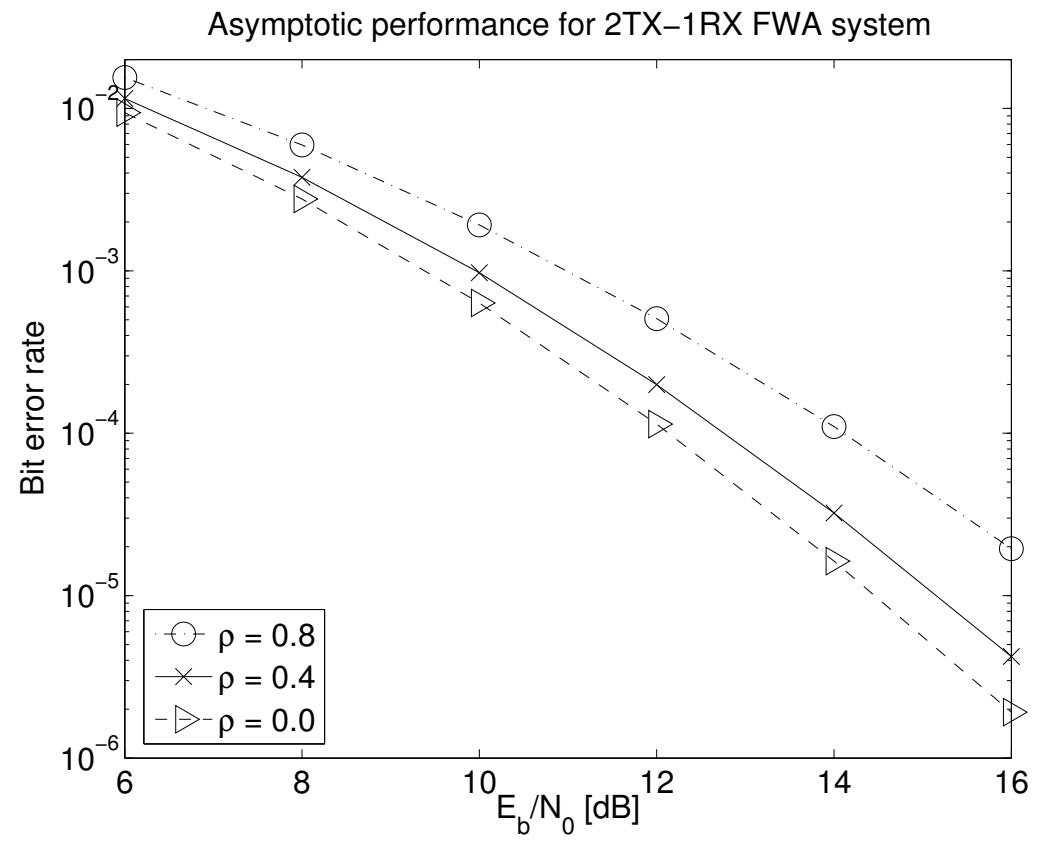

Fig. 5. Impact of antenna correlation on the performance bound for the IC plus 3-path combining scheme. 


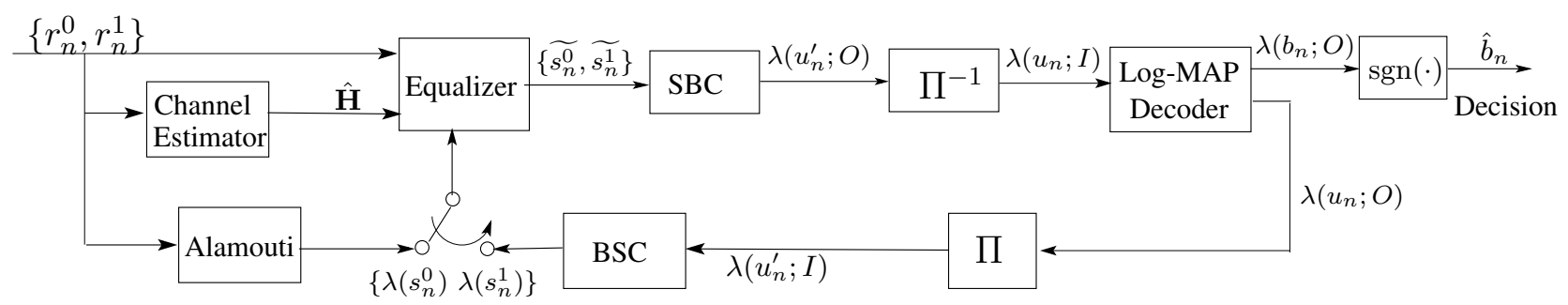

Fig. 6. Diagram of the proposed turbo equalization scheme for the BFWA SUI-3 channel. 


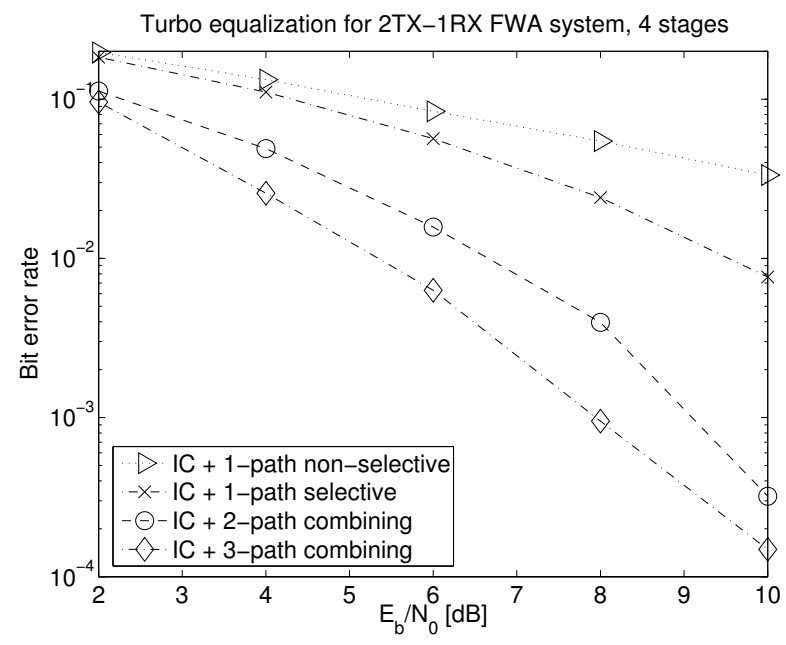

(a) $2 \mathrm{TX}-1 \mathrm{RX}$ System.

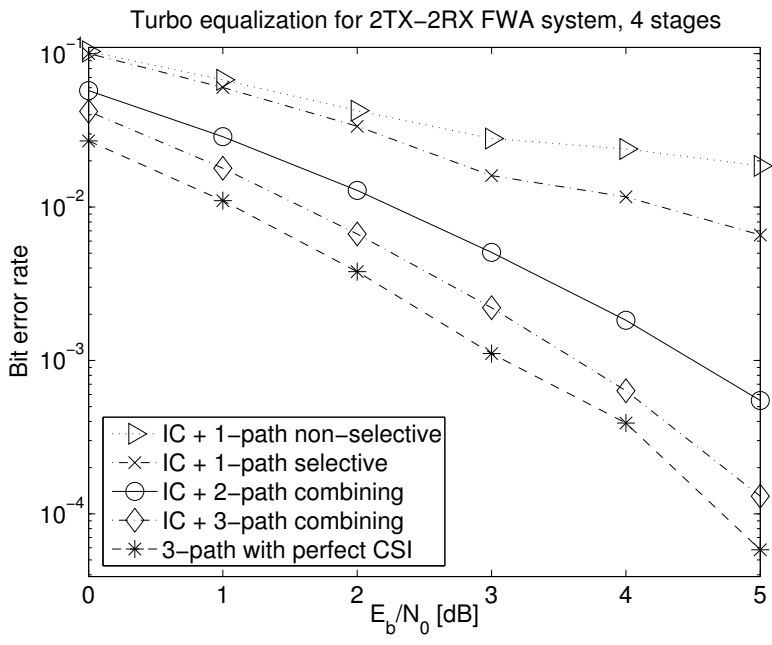

(b) $2 \mathrm{TX}-2 \mathrm{RX}$ System.

Fig. 7. Comparison of different space-time turbo equalization schemes for the STBC BFWA system. The curves represent the 4th stage turbo equalization. 


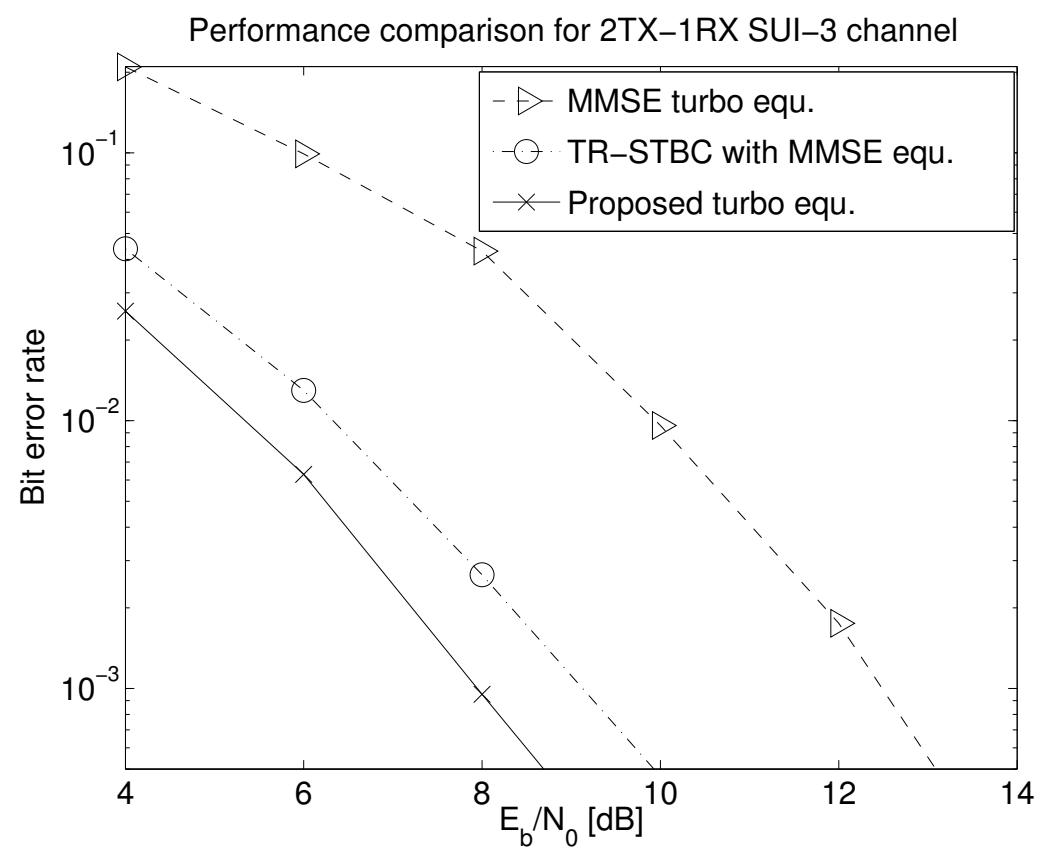

Fig. 8. Comparison of different space-time equalization schemes. 
TABLE I

THE ENCODING AND TRANSMISSION SEQUENCE FOR THE 2 TX ANTENNA SYSTEM.

\begin{tabular}{|c|c|c|c|c|c|c|}
\hline \hline & $t-2 T$ & $t-T$ & $t$ & $t+T$ & $t+2 T$ & $t+3 T$ \\
\hline antenna 0 & $s_{n-1}^{0}$ & $-s_{n-1}^{1 *}$ & $s_{n}^{0}$ & $-s_{n}^{1 *}$ & $s_{n+1}^{0}$ & $-s_{n+1}^{1 *}$ \\
\hline antenna 1 & $s_{n-1}^{1}$ & $s_{n-1}^{0 *}$ & $s_{n}^{1}$ & $s_{n}^{0 *}$ & $s_{n+1}^{1}$ & $s_{n+1}^{0 *}$ \\
\hline
\end{tabular}

